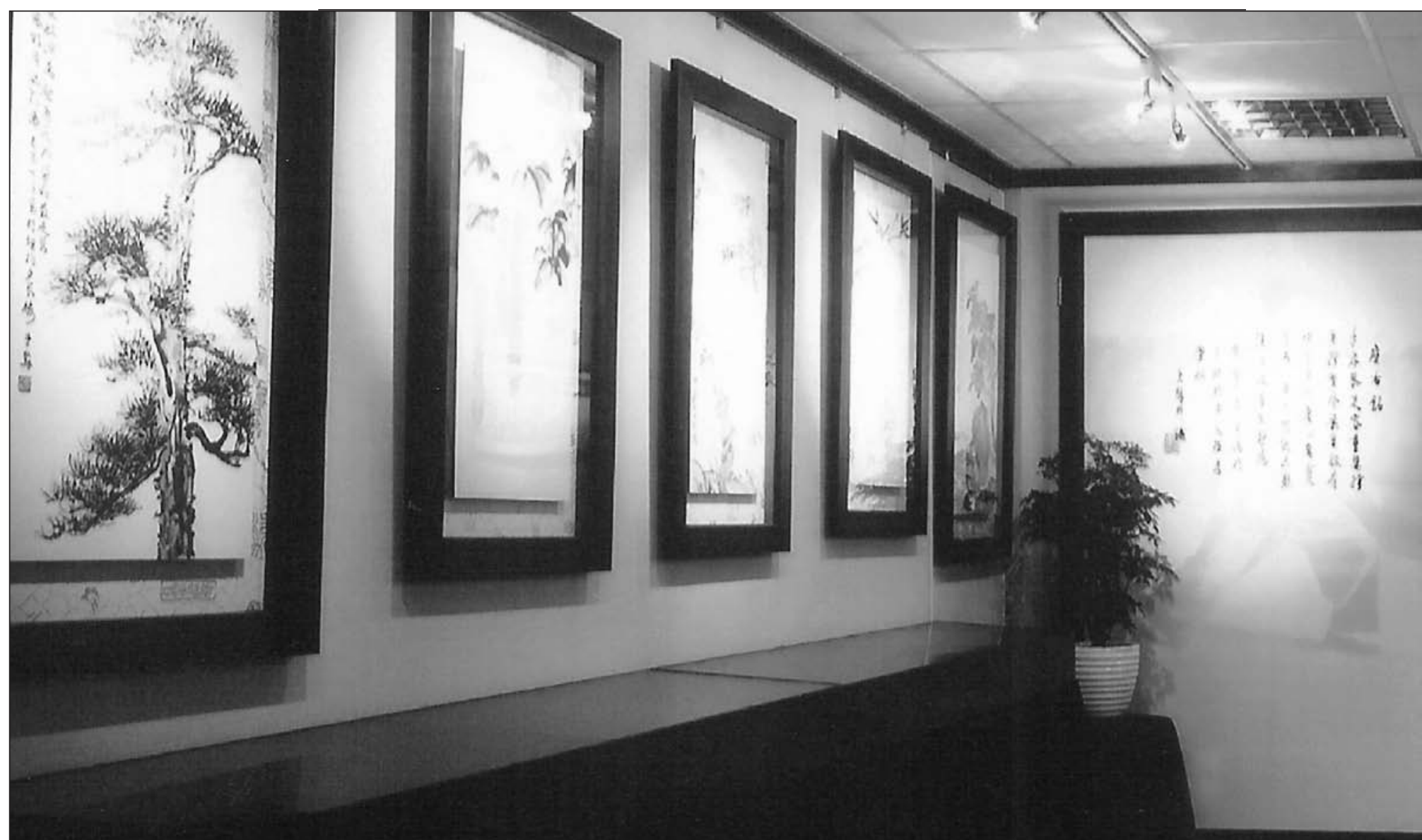

Fotografía interior cortesía de Robert W. Smith

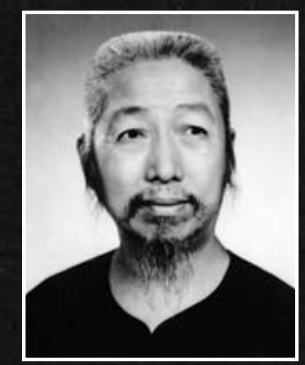

LA GRAN SALA DE

EXPOSICIÓN EN LA

PLANTA BAJA DEL SALÓN

Conmemorativo de

ZHENG MANQING, DONDE SE EXPONEN VARIOS CUADROS Y FotografíAs DEL PROFESOR

ZHENG.

Fotografía cortesía de Yuan Weiming.

\title{
Resumen
}

El profesor Zheng Manqing (1902-1975), un excelente artista y un notable discípulo de taijiquan de Yang Chengfu, emigró a Taiwán en 1949 después de la guerra civil china entre Guomindang y facciones maoístas. Bajo el Movimiento Cultural del Renacimiento del Presidente de la República de China Chiang Kai-shek, Zheng desempeñó un importante papel en la preservación de los tesoros culturales de la China tradicional. Durante el periodo de la Revolución Cultural de Mao Zedong, cuando el continente estaba velado a los ojos extranjeros tras la Cortina de Bambú, Zheng contribuyó decisivamente en la introducción del taijiquan y otros elementos de la cultura China en Occidente. Zheng murió en su casa de Yonghe, Taiwán, en 1975, pero no sin antes cimentar su reputación internacional por su maestría en el taijiquan y en otras artes. Recientemente, una parte de su antigua residencia fue convertida en salón conmemorativo (el Zheng Manqing Jinian Guan) por sus estudiantes más antiguos interesados en preservar su tradición marcial, pinturas, caligrafía, y otros artefactos. Este artículo proporciona una pequeña historia de la vida de Zheng y su legado en Taiwán, así como un recorrido fotográfico del Salón Conmemorativo de Zheng Manqing. 


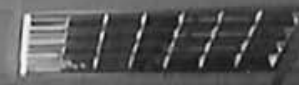

\section{ZHENG MANQING: el Salón Conmemorativo y el legado del Maestro de las Cinco Excelencias EN TAIWÁN}

\author{
Russ MASON
}

\section{Introducción}

El Profesor Zheng Manqing (Cheng Man-ch'ing) ha sido denominado como un "genio taiji" y un "sabio multifacético" (Smith, 1999: 201). Un puente viviente entre la China antigua y la sociedad occidental contemporánea, Zheng encarnó el ideal del caballero chino culto de clase intelectual. En la antigua China, un erudito necesitaba un profundo conocimiento de los clásicos de la literatura, caligrafía, y poesía para superar los exámenes del servicio civil imperial que aún se celebraban durante la infancia de Zheng, a finales de la Dinastía Qing. Estos requisitos del funcionariado público dieron forma al concepto chino de educación. Más allá de estos logros se esperaba que un hombre cultivado estuviera familiarizado con la pintura, el juego de Go (weiqi), y otras artes clásicas (Barnstone y Chou, 1996: xii). Zheng sobresalió en todas estas artes. Además, siguiendo el ideal confuciano, Zheng combinó las artes marciales con las bellas artes, siguiendo las enseñanzas de Confucio de que sin entrenamiento marcial (e.g., tiro con arco y manejo de carros) uno no podría dominar la disciplina mental necesaria para las artes civiles (e.g., decoro, música, matemáticas, y escritura) (Zheng, 1985: 14).

Se conoce al profesor Zheng como el "Maestro de las Cinco Excelencias", refiriéndose a sus sobresalientes aptitudes en medicina tradicional china, artes marciales, y bellas artes (pintura, poesía, y caligrafía). Un investigador americano ha escrito que, para evaluar correctamente los logros de Zheng en el contexto chino, un occidental tiene que comenzar imaginando una persona que ha hecho extraordinarias contribuciones en la erudición clásica, teoría literaria, y crítica. Además, tal persona es: "un boxeador olímpico, presidente de una asociación médica nacional, un poeta distinguido y profesor de literatura, y tiene [una de sus] pinturas colgadas en el Louvre" (Wile, 2007: 2). ¿Cómo cimentó el profesor Zheng una reputación de tal grado como para ser digno de un monumento conmemorativo?

Las siguientes secciones presentan un conciso esbozo biográfico de los primeros años de la vida personal de Zheng, sus éxitos académicos y profesionales, y su entrenamiento y logros en el campo de las artes marciales. Se centrará la atención en su trabajo en Taiwán, el crecimiento de su escuela Taipei, y en el establecimiento póstumo del salón conmemorativo situado actualmente en su antigua residencia. 
EL PROFESOR ZHENG

COMPLETA UN POEMA.

Fotografía cortesía de

Ken van Sickle

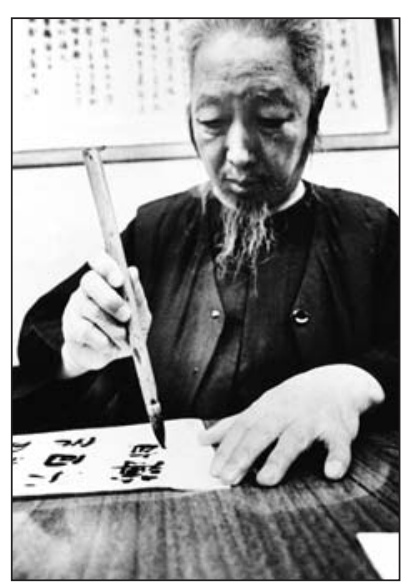

\section{Primeros años}

Nacido en la Comarca de Yongjia (ahora Distrito Lucheng de Wenzhou) en la provincia de Zhejiang, Zheng Yue ${ }^{1}$ creció como el más joven de una modesta familia. Su padre murió cuando era todavía un niño, y su vida fue dura. A pesar de eso, incluso de niño, el interés de Zheng en la caligrafía, pintura, poesía, y hierbas medicinales fue estimulado y educado por su madre y su tía materna. Yue era un niño precoz con un intelecto agudo y curioso que había memorizado los clásicos de Confucio a los nueve años. Luego, tras sufrir una fractura del cráneo en un accidente, pasó dos días en coma. Un profesor de artes marciales trató al chico con hierbas de la montaña, y recobró la consciencia pero había perdido completamente su memoria y estaba en un estado casi vegetativo. Poco después su madre colocó a Zheng como aprendiz de un pintor local, Wang Xiangchan ${ }^{2}$. El trabajo simple, repetitivo, de moler tinta, se probó terapéutico, y en cinco años el joven artista no sólo había recuperado su agudeza mental sino que también había perfeccionado tanto su habilidad para pintar como para empezar a mantener a su familia y a sí mismo vendiendo sus propias obras.

\section{Éxito académico y profesional}

En 1916, el famoso poeta Lu Changbei dio una recomendación al artista en ciernes que le llevó a un mayor estudio en pintura, poesía, y caligrafía en Hangzhou (Wile, 2007: 16). Tres años más tarde, a la tierna de edad de diecisiete años, Zheng viajó a Beijing donde se encontró y entabló amistad con varios pintores y poetas mayores, ganando su respeto debido a su inteligencia y habilidad. A su alrededor, los jóvenes pensadores quedaban enganchados en el movimiento reformista del Cuatro de Mayo y los primeros días de la nueva República estaban cuestionando las formas tradicionales, pero los guanxi (contactos sociales) de Zheng con miembros de las generaciones más viejas, desarrollados por su participación en estos círculos artísticos tradicionales, hizo que sobre 1919 le invitasen a enseñar poesía en la Universidad de Yuwen, Beijing. Poco después, Zheng recibió una recomendación para dar clases en la Universidad Nacional de Jinan en Shanghai de parte de Cai Yuanpei (1868-1940), uno de los educadores de mayor rango y más influyentes de la temprana Era Republicana (Davis, 1996: 40, 53). Cai fue rector de la Universidad de Beijing en esa época. Parece razonable suponer que el apoyo personal de Cai abrió puertas y ayudó a impulsar el rápido crecimiento del joven en el mundo académico.

Después de su traslado al sur desde la capital, Zheng se convirtió en director del departamento de pintura de la Escuela de Shanghai de Bellas Artes y allí también contribuyó decisivamente en el establecimiento del Colegio de Cultura y Arte. Pero sus responsabilidades en Shanghai no le impidieron organizar una exposición individual de arte en el Pabellón de la Rivera (Shuixie) en el parque central de Beijing, y en 1925, junto con colegas como el famoso artista Zhang Daqian, fundar la Sociedad de Pintura Xiaohan (Wile, 2007: 16). También viajó a Japón para investigar las bellas artes para el Ministro de Educación de China (Davis, 1996: 40).

Los estudiantes de Zheng relatan una anécdota ${ }^{3}$ que ilustra claramente la aptitud artística del joven profesor en este momento de su carrera, así como su memoria fotográfica. Una vez, mientras atendía una cena en la casa del alcalde de Shanghai, Zheng quedó fascinado de un manuscrito colgado creado por un famoso maestro de pintura sobre juncos. Separándose de la reunión de los distinguidos invitados, miró el manuscrito durante bastante tiempo, lleno de admiración. Cuando se le sugirió que podría llevarse la pintura para estudiarla en la privacidad de su casa, el joven artista rehusó, diciendo que dicho estudio prolongado era innecesario ya que ya había aprendido la escena de memoria. Al siguiente día Zheng presentó una replica de la pintura a su anfitrión. El alcalde Wu estaba sorprendido al ver que la réplica pintada por Zheng igualaba al original en cada detalle y era, en sí misma, una obra de arte magistral. Después de esto, se propagó la noticia sobre la genialidad artística de Zheng por todo Shanghai. 
Algunas fuentes sugieren que el traslado de Zheng hacia el sur pudo haber estado motivado parcialmente por asuntos de salud, ya que sufría de tuberculosis, que se agravaba por el clima del norte de Beijing así como por el omnipresente polvo de tiza del ambiente del aula ${ }^{4}$. Durante esta época en Shanghai, Zheng comenzó a dedicarse a un estudio más serio de la medicina china. Basándose en el conocimiento de remedios herbales obtenidos de su madre en primer término, Zheng buscó y consiguió recibir enseñanza del Dr. Song You'an, un famoso practicante de la provincia de Anhui que fue convencido para dejar la jubilación y aceptar un aprendiz personal. Mucho más tarde, Zheng ejerció a tiempo completo la medicina herbal, siendo finalmente presidente de la Asociación Médica Nacional China (Davis, 1996: 41). Además de ser nombrado para la Asamblea Nacional para la Construcción de la Constitución en 1946, Zheng fue elegido un año después por la Asamblea Nacional para representar a la comunidad de doctores de Medicina Tradicional China, una posición que ostentó hasta el final de su vida (Cheng, 1971: 238).

Zheng Manqing era ampliamente reconocido como un hombre de grandes y variados logros, tanto que Lin Sen (1868-1943), el Presidente del gobierno nacionalista en la época de la Guerra Chino-Japonesa, entregó a Zheng una inscripción caligráfica concediéndole el título de "Maestro de las Cinco Artes".

A la edad de cuarenta años, Zheng se casó con Ding Yidu (conocida en los Estados Unidos como Madame Juliana T. Cheng), la hija del general Ding Muhan (fundador de la Oficina de la Fuerza Aérea China). Madame Zheng había sido una estudiante de medicina en la Universidad de Beijing, y su especialidad en la práctica médica fue la obstetricia. La pareja tuvo cinco hijos.

\section{Logros marciales}

Quizá debido a sus problemas de salud en la infancia, en sus primeros años Zheng estudió prácticas tales como el baduanjin y el yijinjing (Davis, 1996: 40). También practicó taijiquan de manera intermitente desde 1923 en un intento por reforzar su débil físico, pero cada vez que mejoraba su condición física, suspendía la práctica para dedicarse a otros esfuerzos ${ }^{6}$. Después de que la tuberculosis de Zheng progresase hasta el punto de llegar a expectorar sangre, se comprometió a perseverar en un régimen de ejercicio diario. Zheng obtuvo una recomendación para el

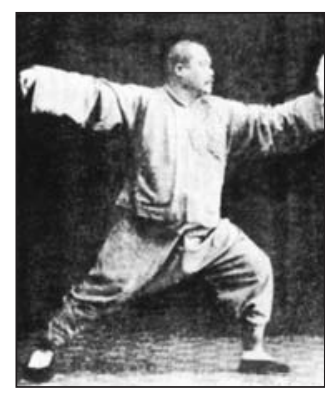
famoso maestro Yang Chengfu y acreditó la valía de la práctica del taijiquan Estilo Yang con su completa recuperación de la enfermedad que amenazaba su vida (Cheng, 1971: 238; Cheng, 1985: 64; Yang, 1934/2005: 2).

En su propio libro, Yang Zhenji (el segundo hijo de Yang Chengfu; nacido en 1921) enfatizó el hecho de que Zheng era el discípulo de su padre y que Zheng era el sabio que tradujo el libro de Yang Chengfu de 1934, La esencia y aplicaciones del Taijiquan (Taijiquan tiyong quanshu) (Yang, 1993: 250). Además de ser escogido para registrar las enseñanzas de Yang, Zheng fue honrado al pedírsele que escribiera el prefacio para el libro del maestro?.

Uno de los estudiantes más antiguos de Yang Chengfu fue Li Yaxuan. El estudiante de Li, Yan Changkong, escribe que después de la victoria maoísta, las posteriores ediciones del libro de Yang censuraban el nombre de Zheng y su prefacio por razones políticas. Esto fue debido a sus conexiones con Chiang Kai-shek. Sin embargo, después de treinta años de bloqueo informativo, el nombre de Zheng y su biografía están apareciendo una vez más en las publicaciones de la China Continental (Wile, 2007: 15, 32; Davis, 1996: 48). Yan también registró la afirmación de Li de que Yang dio instrucción privada a Zheng en el cultivo interno (neigong), que le ayudó a comprender los elementos más sutiles del arte. Yan, citando los altos principios de Li Yaxuan, empezó a notar que las

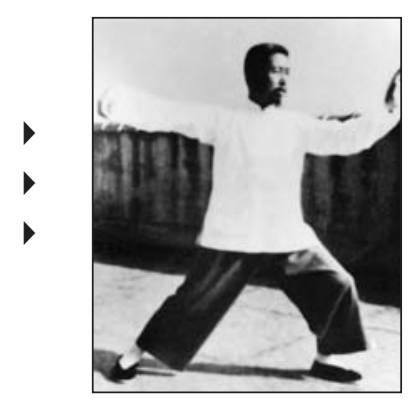

YANG CHENGFu (CA. 1933) Y SU ESTUDIANTE ZHENG MANQING (CA. 1948) MUESTRAN LA POSTURA LÁtigo Simple. 


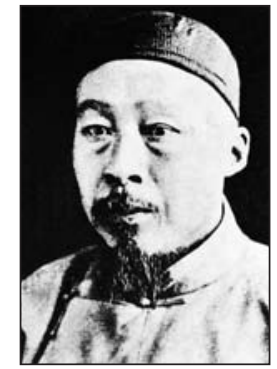

Chen Weiming:

ESTUDIANTE VETERANO DE YANG Chengfu Y COMPAÑERO DE CLASE, AMIGO Y VALEDOR DE

ZHENG MANQING.

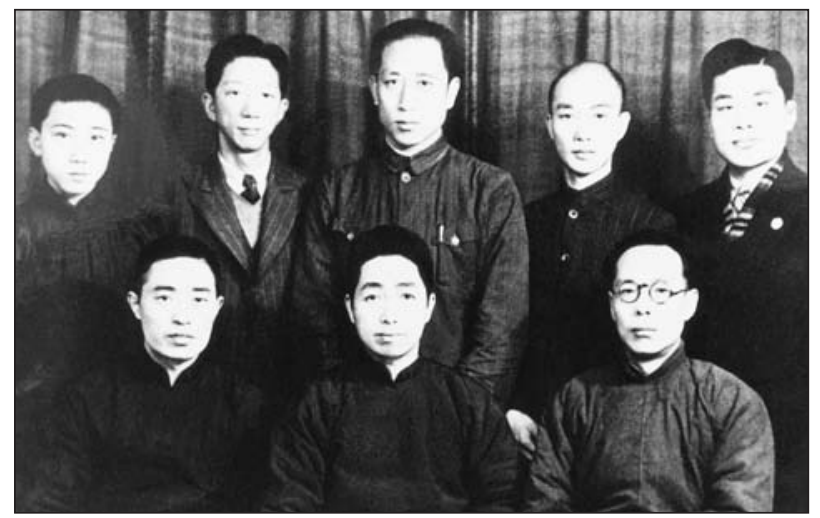

El Profesor Zheng MANQING (CENTRO DE LA FILA DELANTERA) EN China eN 1943 CON UN GRUPO DE ESTUDIANTES: DIRECTAMENTE DETRÁS DE ZHENG está ZHANG ZIGANG; EN LA FILA DE ATRÁS, EN EL EXTREMO DERECHO Y CON PAÑUELO,

ESTÁ GuO QINFANG, CAMPEÓN DE ChINA DEL PESO LIGERO DEL BOXEO OCCIDENTAL A FINALES DE LOS AÑOS 1930.

Fotografía cortesía de Guo Qingfang.

\section{Llegada a Taiwán}

alabanzas de Li por la perspicacia y habilidades de Zheng eran "verdaderamente una cosa extraña" (Wile, 2007: 33, 35).

Otro de los famosos estudiantes de Yang fue Chen Weiming (1881-1958). Chen no sólo era un antiguo estudiante de Yang Chengfu, sino también un erudito y escritor que había representado las enseñanzas de Yang en texto impreso ${ }^{8}$. Después de la Guerra Chino-Japonesa, Zheng llevó el temprano manuscrito de su propio texto, Trece Tratados sobre el Taijiquan del Maestro Zheng, a Chen para ver si contaba con la aprobación de su veterano compañero de clase (Cheng, 1965/1999: 9). Zheng sentía que tanto su libro como su enfoque del taijiquan eran simplemente una continuación de las propias enseñanzas de Yang y de su primer texto, Esencia y aplicaciones del Taijiquan. Chen aprobó el trabajo de Zheng y animó a su publicación, incluso ofreciéndose para escribir un prefacio (Cheng, 1985: 108; Cheng, 1965/1999: 9). Chen escribió en su prefacio del libro que, durante los años de estudio de Zheng, cuando Madame Yang (de soltera Hou) cayó enferma, Zheng utilizó su conocimiento de la medicina herbal para tratarla, salvando su vida. Chen escribe: "El Maestro Yang estaba tan agradecido que enseñó [a Zheng] todas las transmisiones orales secretas. Nadie más las había escuchado hasta entonces" (Wile, 1985: 1). Chen apoyó la creencia de Zheng de que un principio general del Taijiquan Estilo Yang es el compromiso con un doble principio: hundir-relajar (chen song), algo que llegaría a ser una característica distintiva del enfoque del taijiquan de Zheng9.

Fu Zhongwen (1903-1994, nieto, discípulo, y profesor ayudante de Yang Chengfu) declaró que Zheng estableció su reputación en los años 30 en el círculo de Yang debido a su profundo interés en el tuishou (empuje de manos) y su entusiasta entrenamiento con los estudiantes veteranos más habilidosos (Yu \& Sharp, 1993: 45-46). Tras perfeccionar su habilidad trabajando con Yang y sus estudiantes más antiguos, Zheng continuó con varios desafíos públicos y privados, algunos implicando a personal militar británico y americano y otros con boxeadores chinos muy respetados. El hecho de que Zheng sirviese como director en la Academia de Artes Marciales de Hunan (así como en la facultad de otras dos academias militares), y el hecho de que se ganase el respeto de los oficiales militares experimentados con los que trabajó, atestiguan la funcionalidad de sus habilidades ${ }^{10}$.

En el continente, se pidió la ayuda de Zheng en 1933 para enseñar taijiquan a las tropas Nacionalistas en la Academia Central Militar (antiguamente conocida como Huangpu). Más tarde, durante la Guerra Chino-Japonesa, dio clases en Sichuan para el Grupo de Entrenamiento Militar Central (Davis, 1996: 41). En 1938, como director de la Academia de Artes Marciales de Hunan del gobierno provincial, Zheng fue responsable del entrenamiento de centenares de oficiales en tan sólo dos meses. Bajo estas circunstancias fue forzado a simplificar la forma larga Yang, eliminando repeticiones, estableciendo finalmente 37 como el número habitual de posturas para su versión del ejercicio individual ${ }^{11}$.

Después de la rendición de Japón en 1945, volvió a estallar una guerra civil a gran escala en China entre las fuerzas Nacionalistas del Generalísimo Chiang Kai-shek (el Guomindang) y los Maoístas. Después de la victoria comunista en el continente en 1949, Zheng emigró a Taiwán con el Guomindang. Finalmente, se instaló en una casa en la carretera Zhongxing en Yunghe, un suburbio de la capital de Chiang Kai-shek, Taipei. Apodaría al lugar Xi Chang Lou, lo que podría traducirse como "Torre de la Larga Tarde" o "Posada de la Larga Noche" (Wile, 2007: 52). Allí, se concentró en practicar la medicina, abriendo más tarde una escuela de taijiquan en la planta superior del Salón Zhongshan a petición del alcalde 
de Taipei, You Mijian (Wile, 2007: 18). En Taiwán, Zheng enseñó su forma "Simplificada" de 37 posturas (Jianyi taijiquan) públicamente a civiles.

Después de establecerse como médico en Taiwán, Zheng aceptó a su primer estudiante del arte del taijiquan en la isla. Benjamín P.J. Lo, un joven universitario, llegó para un tratamiento médico y se le aconsejó practicar taijiquan para que su cuerpo pudiese desarrollar la fuerza necesaria para absorber la medicación herbal prescrita (Davis y Mann, 1996: 50) ${ }^{12}$. Comenzando en 1949, Lo fue a la casa del Profesor Zheng cada día para aprender taijiquan de la forma tradicional, perfeccionando la forma postura a postura. Luego fue a los Estados Unidos donde ha enseñado el método Zheng desde 1974. Poco después de que Lo comenzase a estudiar, Liu Xiheng se presentó como discípulo a Zheng, seguido por Xiu Yizhong y otros. A medida que la fama del maestro se extendió por toda la isla, muchos más estudiantes se fueron sumando, la mayoría habiendo desarrollado un entrenamiento previo en las artes marciales y, en la forma tradicional, por recomendación personal. Zheng fundó la Sociedad de Estudio Shi Zhong (Shi Zhong Xueshe) $)^{13}$ en 1949 para el fomento del taijiquan (Davis, 1996: 43; Wile, 2007: 7; véase también la página web de Shi Zhong).

Además de dirigir su propia escuela, la Sociedad de Estudio Shi Zhong, Zheng fue miembro del Club Chino de Taijiquan (Zhong Guo Taijiquan Ju Le Bu), fundado oficialmente el 27 de marzo de 1960. El miembro de la Asamblea Nacional, Chen Panling (que también era director de la Asociación China de Boxeo R.O.C.) sirvió como comisario jefe. Además de Zheng y Chen, participaban otros miembros muy conocidos incluyendo a Wang Llenan, Xiong Yangho, Guo Lianyin, y Han Qingtang. Más tarde, en 1963, una versión ampliada del club, conocida como el Comité de Investigación Académica del Taijiquan, fue fundada bajo los auspicios de la Asociación Cultural y Económica ChinoAmericana, con Zheng actuando como asesor (Fairchild y Lin, 2007: 2-3).

Durante el periodo comprendido entre 1959 y 1962, el historiador americano de las artes marciales Robert W. Smith fue destinado a una oficina de la CIA en Taiwán como consultor de inteligencia del almirante de la Flota Estadounidense del Pacífico. Su investigación en la práctica e historia de las artes marciales chinas le guiaron a la puerta de Zheng y, finalmente, a una entrega de por vida al método de taijiquan de Zheng ${ }^{14}$. Como primer estudiante occidental de Zheng, Smith hizo muchísimo por divulgar a "el Profesor" (como era llamado con respeto por sus estudiantes) en Occidente. En 1964, Zheng viajó al extranjero, primero a París y luego a los Estados Unidos, donde mostró sus pinturas en el Museo de Arte Chino Cernuschi y en el Pabellón de la República China de la Feria Mundial de Nueva York, respectivamente ${ }^{15}$.

Después de establecer su residencia en la ciudad de Nueva York, Zheng recibió el titulo de "Director de Bellas Artes, Movimiento de Renacimiento Cultural de la República de China, Sección Americana" del gobierno de Chiang Kai-shek (Davis, 1996: 46). De este modo, fundó una sección americana de su escuela Shi Zhong para fomentar el taijiquan, así como otras formas de las artes y cultura chinas tradicionales. Dejando a Liu Xiheng al cargo de su escuela en Taiwán en su ausencia (Davis, 1996: 34), Zheng repartió su tiempo entre sus casas de Nueva York y Taipei durante los siguientes diez años. Durante este periodo exhibió pinturas en la Galería FAR en 1968 y en el Museo del Río Hudson en 1973 y escribió algunas de sus más profundas obras, incluyendo comentarios sobre el Daodejing, el Yijing, y los clásicos confucianos de los Analectas, el Gran Aprendizaje, y la Doctrina del Medio (Cheng, 1971/1981: 239; Davis, 1996: 46). En este momento de su vida, Zheng dirigió las escuelas de taijiquan que florecían tanto en América como en la República de China. Su trabajo hizo mucho para popularizar el taijiquan en Occidente y Taiwán y para establecer la reputación internacional de su Forma Corta de 37 posturas del Estilo Yang Simplificado. El profesor Zheng fue especialmente famoso en la comunidad internacional de artes marciales por su asombrosa habilidad en el empuje de manos (tuishou). 


\section{Zheng Manqing \\ (1902-1975).}

A LA DERECHA: EL PROFESOR

ZHENG OBSERVA A ESTUDIANTES SINTIENDO LAS

MANOS EN SU RESIDENCIA EN Yonghe.

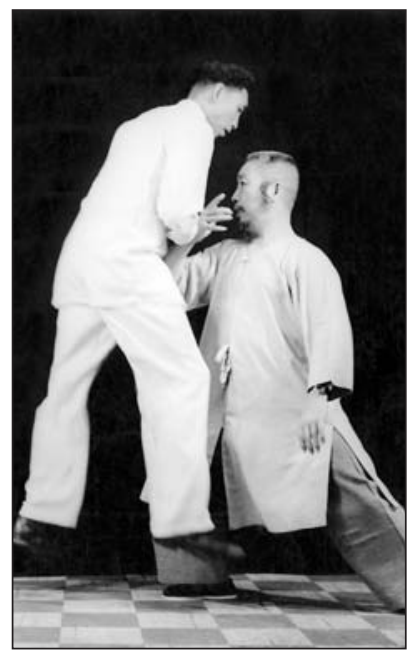

ZHENG DESENRAízA A

William C.C. Chen;

OBSERVE LAS MANOS

RELAJADAS Y EL EFECTIVO

TIFANG (LITERALMENTE:

LEVANTAR/DEJAR-IR).
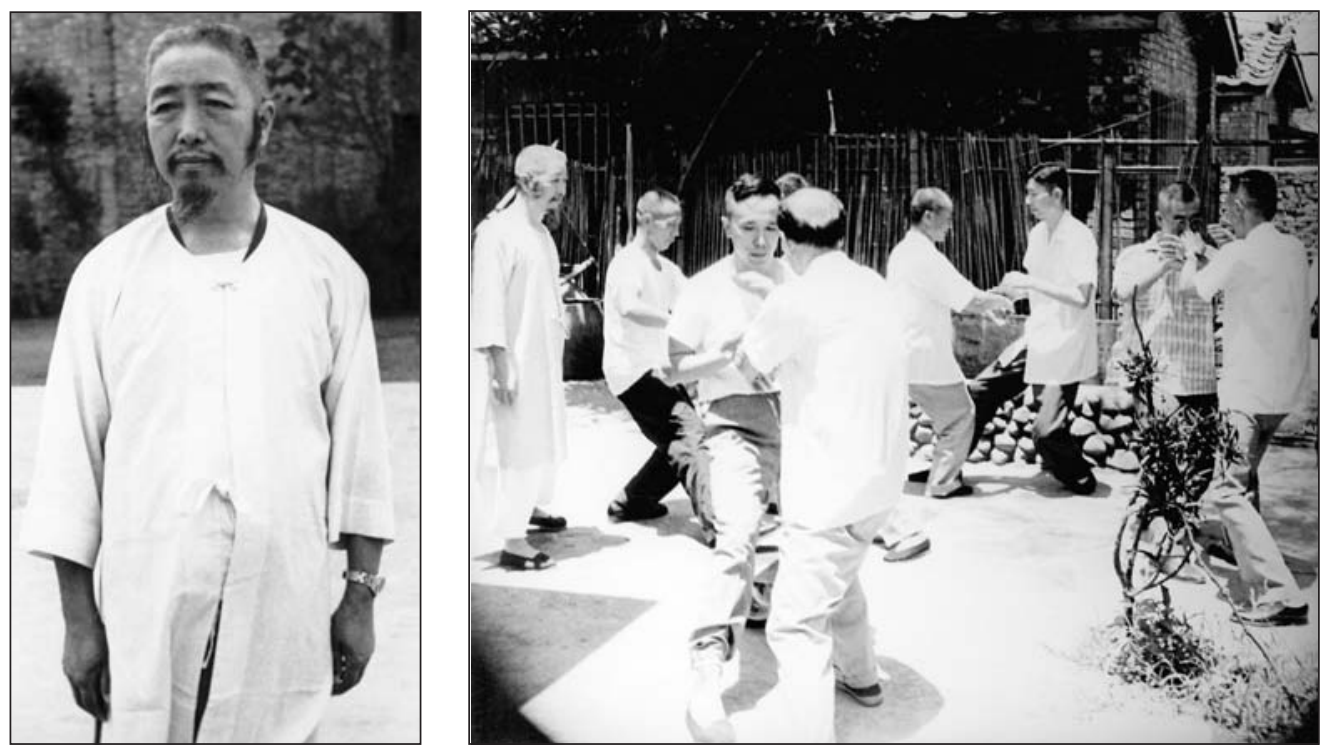

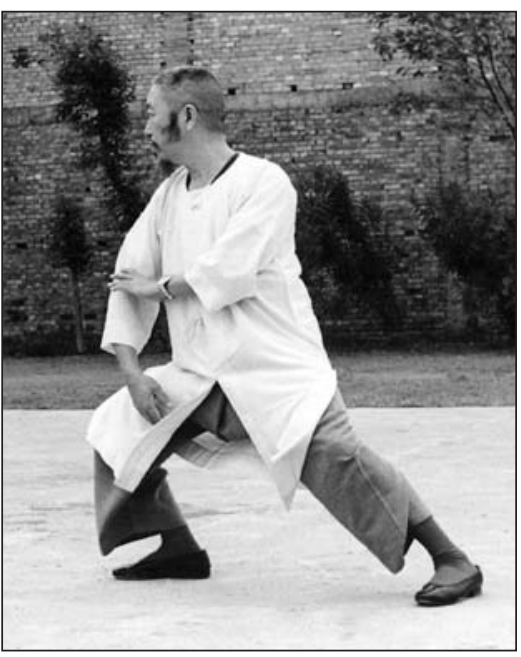

En la postura Golpe de Hombro

Derecha, Zheng RESISTE EL EMPUJE

DE CUATRO HOMBRES (TAIWÁN, APROXIMADAMENTE EN 1960)

IZQUIERDA, ZHENG MUESTRA LA POSTURA LÁTigo Simple Agachándose.

Todas las fotografias de esta página son cortesía de Robert W. Smith.

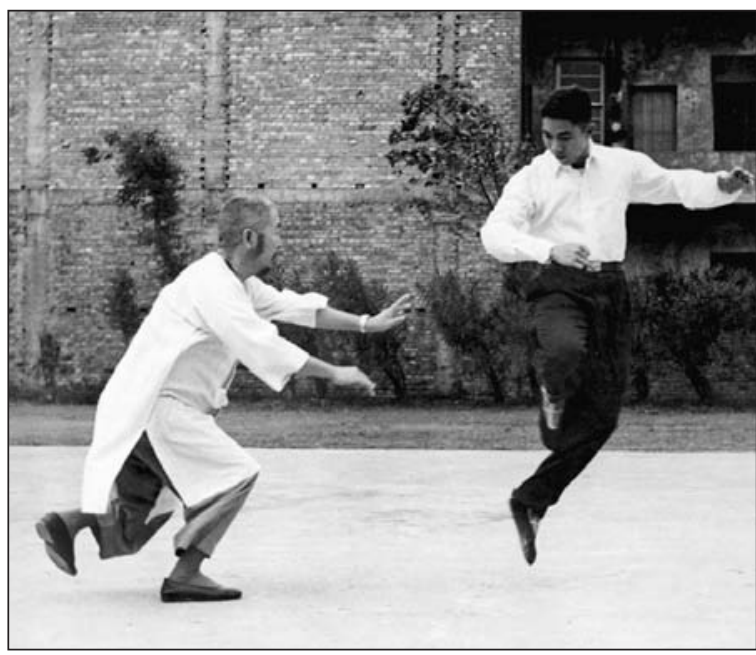

REPELIENDO A UN ATACANTE; OBSERVE EL IMPORTANTE EMPUJE CRUZADO: ENRAIZADO EL PIE DERECHO Y LA MANO IZQUIERDA ACTIVA.

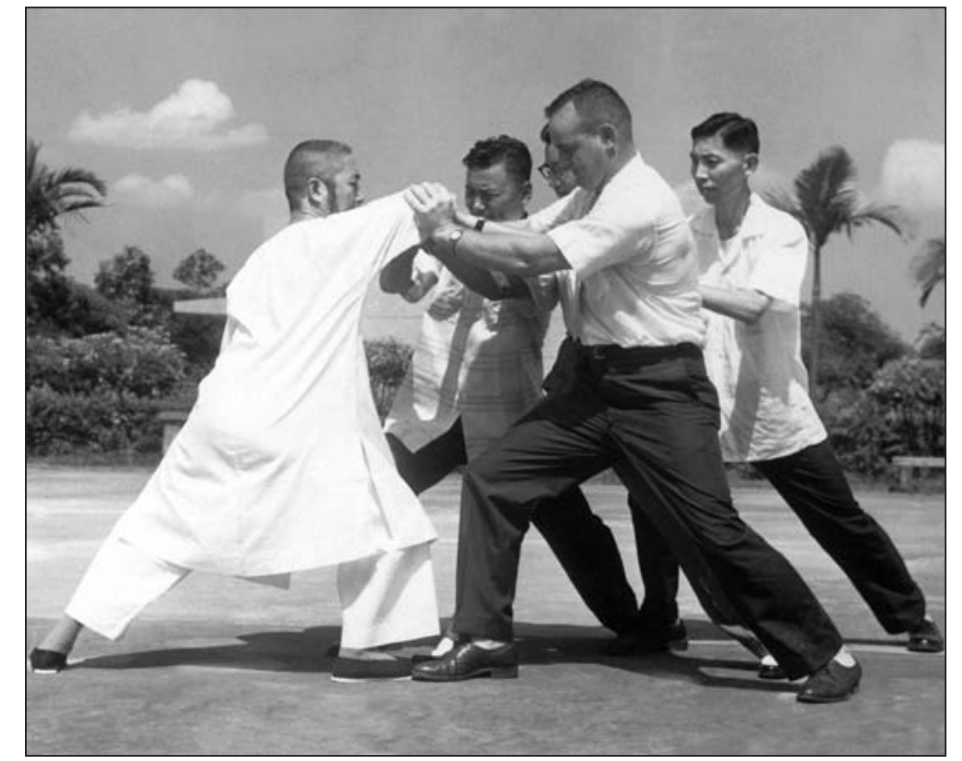


La muerte y legado de Zheng en Taiwán

Zheng murió el 26 de marzo de 1973 debido a los efectos de una hemorragia cerebral sufrida en su casa de Taipei (Wile, 2007: 19). Al funeral del Maestro de las Cinco Excelencias acudieron cientos de personas, incluyendo distinguidos representantes de sus muchos ámbitos de interés, estudiantes, y oficiales gubernamentales. También se levantaron monumentos conmemorativos en Singapur y en la ciudad de Nueva York (Davis, 1996: 48).

Tras la muerte del profesor Zheng, Liu Xihong le sucedió como presidente de la Sociedad de Estudio Shi Zhong de Taipei en 1975, dirigiendo la instrucción del taijiquan en la escuela hasta su jubilación en 1986. Su compañero de clase, Xu Yizhong, ha servido como presidente de la organización desde ese momento. Además, Xu fue elegido en 1993 Presidente del Primer Comité de una nueva organización, la Asociación para el Estudio del Taijiquan del Maestro Zheng. Xu atendió los dos primeros mandatos en este comité. Ke Chihua sirvió como Presidente del Tercer Comité desde 1999 hasta su muerte en 2001, con Ju Hongbin de Gaoxiong terminando su mandato ${ }^{16}$. Después de esto, Xu Yizhong regresó a la oficina y aún continúa desempeñando este cargo actualmente. Los actuales consultores de la Asociación incluyen dos nombres que serán familiares a la comunidad americana de taijiquan: Benjamín P.J. Lo y William C.C. Chen. Con la ayuda de sus capacitados consultores e instructores, el Presidente de la Asociación, Xu Yizhong, trabaja incansablemente para fomentar el Taijiquan Simplificado del Maestro Zheng en Taiwán y por todo el mundo.

Entre los acontecimientos especiales organizados por la Asociación se celebró el Centenario del nacimiento del Profesor Zheng Manqing. Este acontecimiento, de una semana de duración, fue celebrado en agosto de 2000, realizándose mesas redondas, demostraciones de artes marciales, un torneo, y una exhibición de las pinturas y caligrafías de Zheng cedidas por el Museo del Palacio Nacional ${ }^{17}$.

Hoy en día, el Taijiquan Simplificado ha vuelto al punto de partida, ya que uno de los antiguos estudiantes de Zheng en Taiwán, el Presidente Xu Yizhong, viajó en octubre de 2006 a la Universidad de Nankai en Tianjin en la China Continental para enseñar la forma a petición de la comunidad de artes marciales (wushu) de esa Universidad. El currículo de la Universidad de Nankai ofrece en la actualidad un curso para investigar el Taijiquan Simplificado del Profesor Zheng, y otros programas similares están en fase de discusión. Además, la Universidad de Educación Física de Beijing y otras organizaciones relacionadas han mostrado su interés en publicar en el continente los libros de instrucción de taijiquan de Zheng $^{18}$.

Empuje de manos de LiU Xiheng y Robert

W. Smith en TAipei (1983).

Fotografía cortesía de Robert W. Smith.

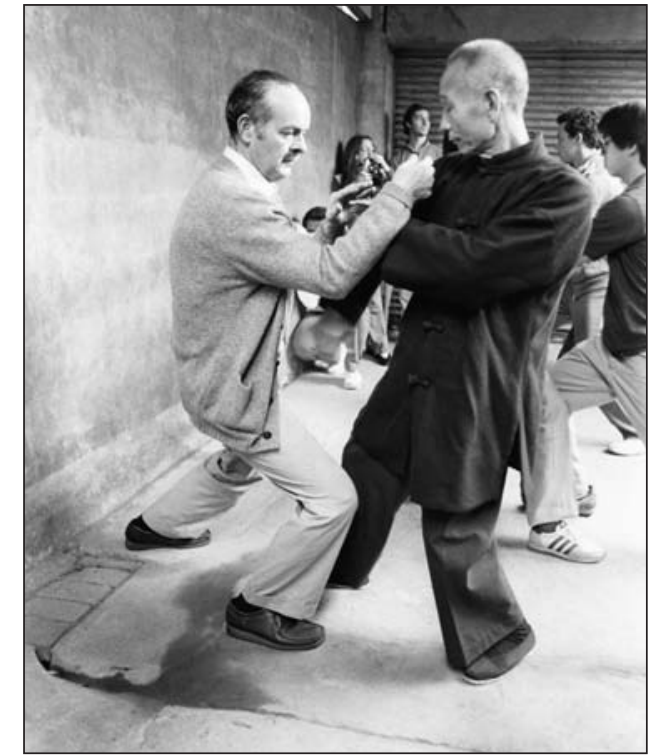

ZHENG CON AMIGOS Y ANTIGUOS ESTUDIANTES.

PRIMERA FILA, DE

IZQUIERDA A DERECHA:

ZHENG; Mr. HONG, AMIGO

Y CONSEJERO LEGAL EN

Hong Kong; Wang

YANNIAN. FILA DE ATRÁS,

DE IZQUIERDA A DERECHA:

Mr. YANG (UN AMIGO

ABOGADO), LIU XIHENG

(PRIMER PRESIDENTE

DE LA ASOCIACIÓN), EL DIFUNTO TAO BINGXIANG (FALLECIMIENTO 2006), Y XU YIZHONG (ACTUAL PRESIDENTE DE LA

ASOCIACIÓN). 


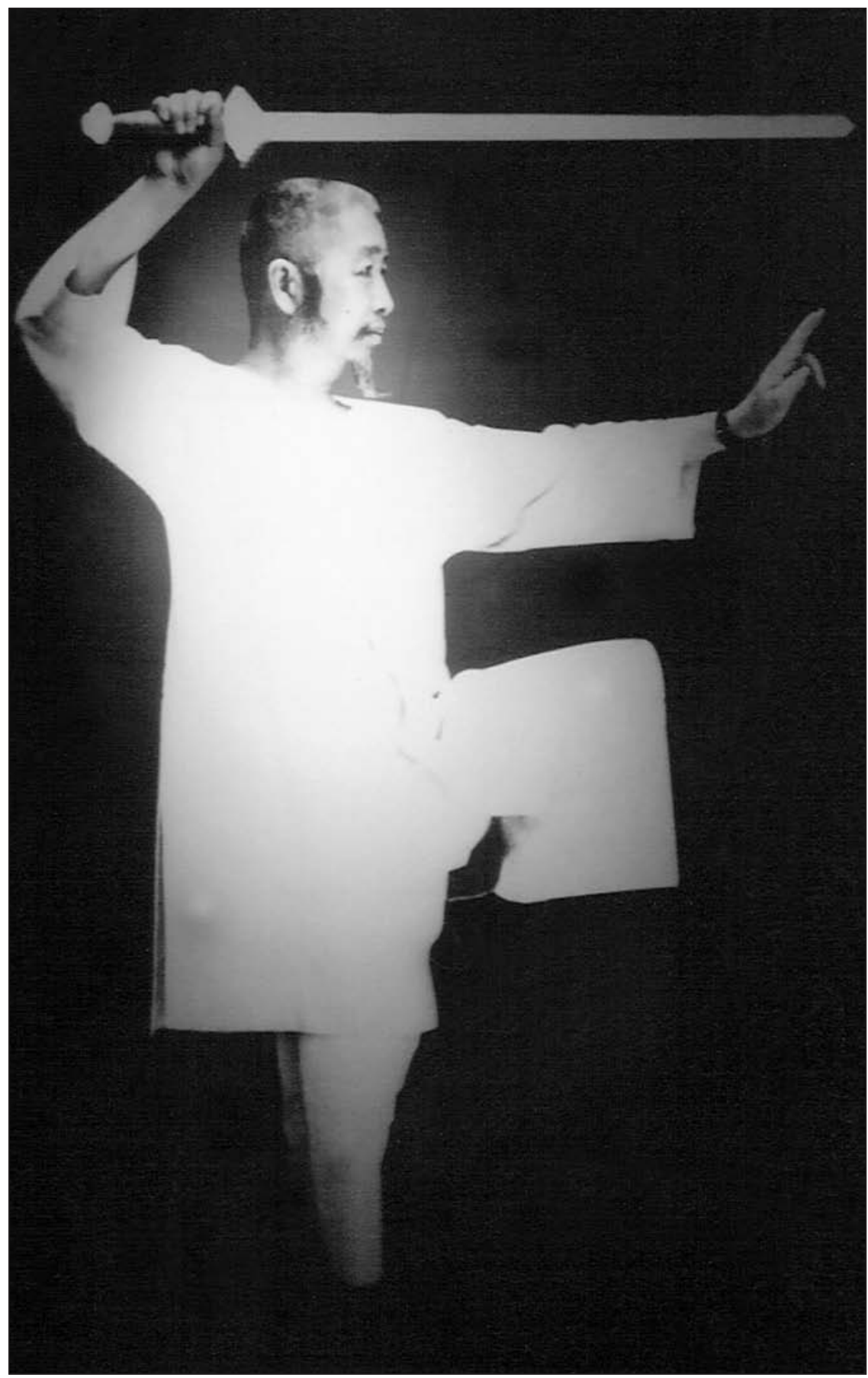

En los años siguientes a la muerte del Profesor Zheng, Madame Juliana T. Zheng y su familia continuaron dividiendo su tiempo entre los Estados Unidos y Taiwán, utilizando la vieja casa en la Calle Zhongxing en Yonghe como base durante sus visitas a Taipei. Después de la muerte de Madame Zheng en enero de 2005, se decidió que una parte de la residencia familiar sería restaurada y convertida en un salón conmemorativo para la preservación de los artefactos y el legado del Profesor Zheng. Después de mucho trabajo por parte de la familia, estudiantes, del Presidente Xu Yizhong, y de la Asociación, la ceremonia de apertura del Salón Conmemorativo de Zheng Manqing se celebró el 25 de junio de 2006. Debido a las limitaciones de espacio y al número de invitados, la ceremonia tuvo lugar en el gimnasio de la Escuela Intermedia Wuchang de Taipei (la localización actual de las clases de taijiquan ofrecidas por la Sociedad de Estudio Shi Zhong). Asistieron quinientas personas, incluyendo destacados artistas, poetas, filósofos, y el director de la Asociación Nacional de Taijiquan de Taiwán, Zhan Deshen. Xu Chongming (el Primer Consultor Presidente de la Asociación para el Estudio del Taijiquan Zhengzi) presentó un informe sobre la historia del Maestro Zheng en Taiwán, y Chou Bozo (el Vice-Presidente del Comité) regaló una Llave de Oro al nuevo salón conmemorativo ${ }^{19}$.

En noviembre de 2006, el que escribe este artículo visitó el Salón Conmemorativo. La escueta visita
Zheng CON LA ESPADA EN la postura Osa Mayor o Estrella PRINCIPAL (DA KUI XING). fotográfica del Salón Conmemorativo Zheng Manqing que aquí se presenta cuenta con el permiso del Presidente Xu y la ayuda del personal de la Asociación y otras personas. Los visitantes del Salón son bienvenidos y pueden recibir más información visitando la página web http://www.37taichi.org.tw/.

Además, se están considerando proyectos para ofrecer suscripciones para la Asociación en el extranjero. Para obtener más información sobre cómo convertirse en miembro del salón conmemorativo, no dude en contactar con la Asociación en 7taichi@ yahoo.com.tw. 

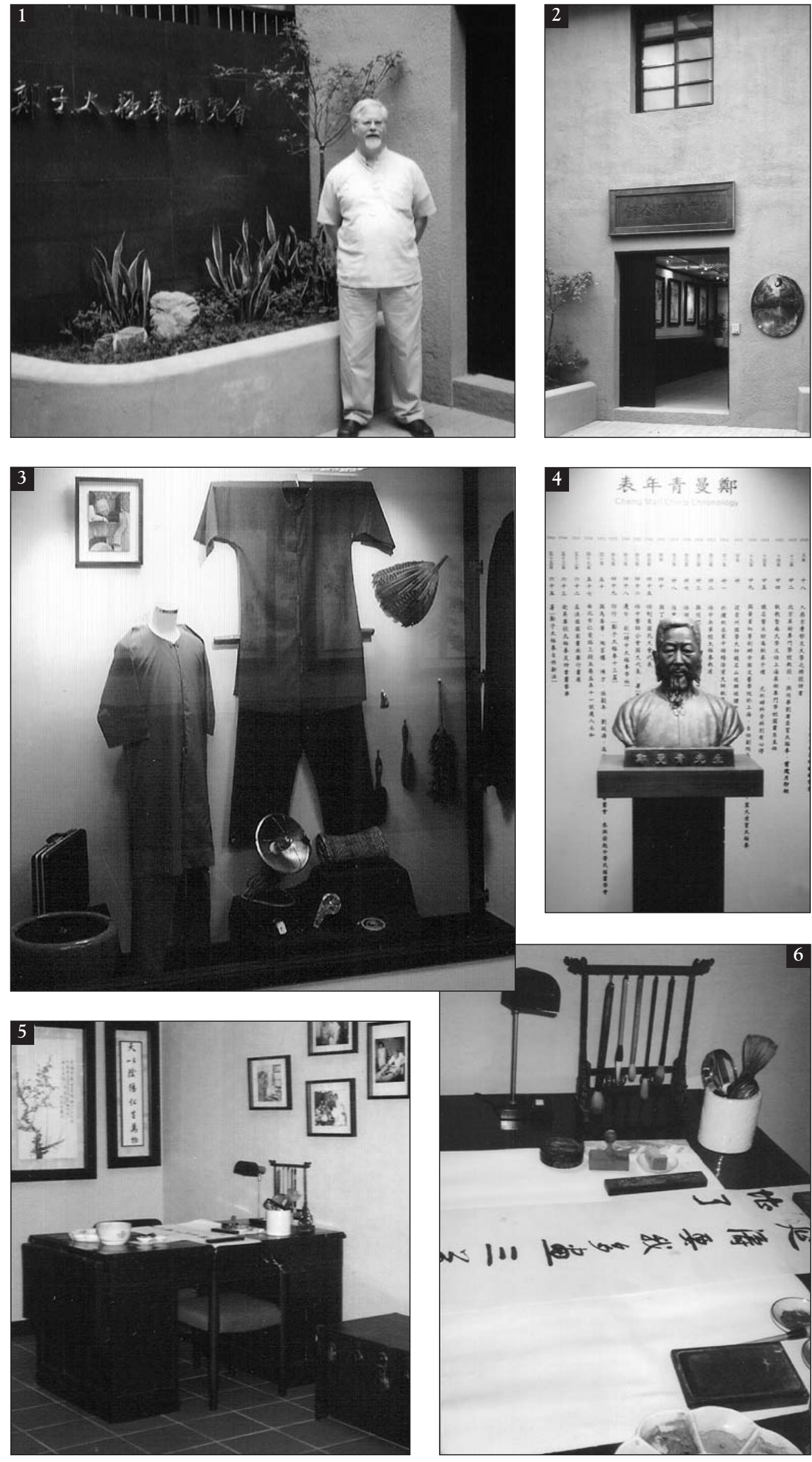

Revista de Artes Marciales Asiáticas $\diamond$ Volumen 3 Número 4 (34-51) - 2008
1) DANNy EMERICK FRENTE A LA PUERTA DEL SALÓN CONMEMORATIVO EN LA ANTIGUAA RESIDENCIA DEL PROFESOR.

Fotografía de R. Mason.

2) LA ENTRADA AL SALÓN CONMEMORATIVO. EL LETRERO ENCIMA DE LA PUERTA DICE: SALÓN CONMEMORATivo Zheng MANQING (ZHENG MANQING Ji Nien GUAN). Fotografía de R. Mason.

3) UNA VITRINA CONTENIENDO LOS ARTEFACTOS PERSONALES DE ZHENG. JUNTO A ÉSTOS TAMBIÉN HAY UN BREVE ÁRBOL GENEALÓGICO Y UNA CRONOLOGÍA DE SU VIDA. Fotografía cortesía de Yuan Weiming.

4) El busto en bronce A TAMAÑO NATURAL DEL PROFESOR ZHENG ESTÁ COLOCADO EN UNA PEQUEÑA ALCOBA CON UNA CRONOLOGÍA DE SUS ACONTECIMIENTOS VITALES MÁS IMPORTANTES.

Fotografía de R. Mason.

5-6) ESCRITORIO DE ZHENG, Y DETALLES DEL ESCRITORIO MOSTRANDO PIEDRA DE TINTA, PINCELES, CALIGRAFÍA Y SELLO PERSONAL.

Fotografías de R. Mason. 


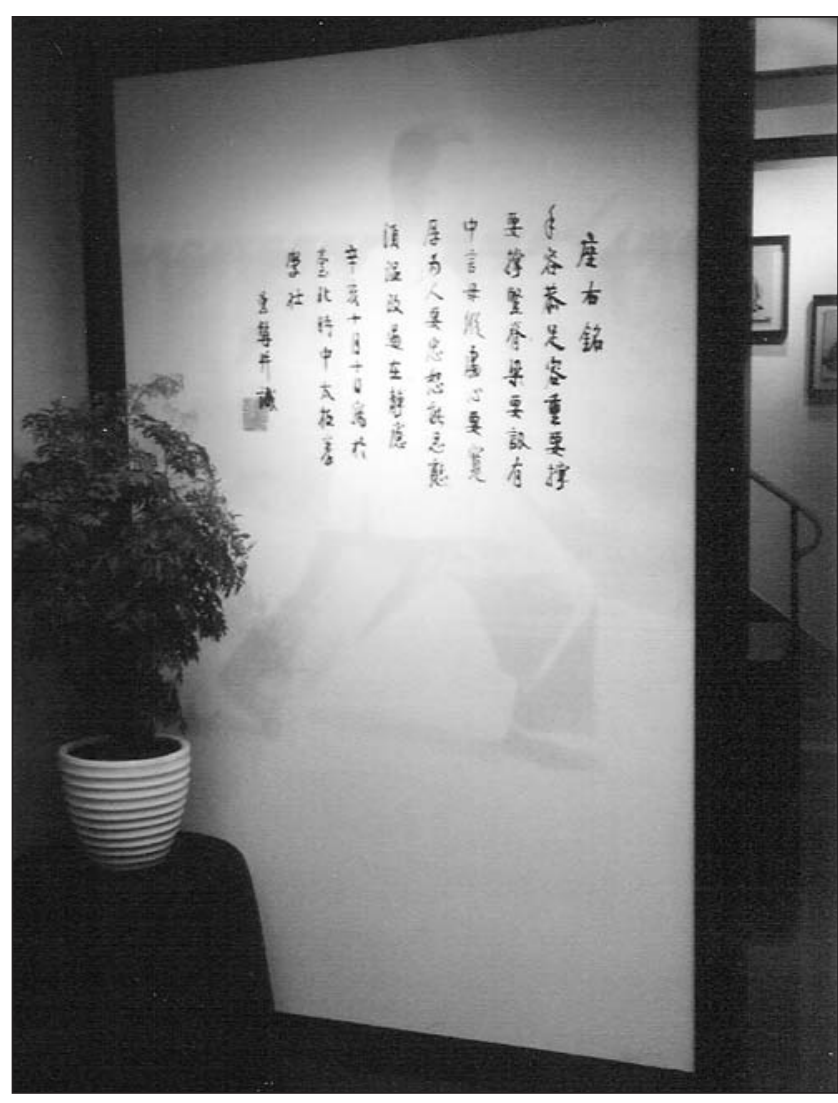

Arriba: la CAligrafía ZuO You Ming DE ZHENG SUPERPUESTA EN UNA FOTO SUYA EN LA POSTURA DE LÁtigo Simple. DERECHA: MANUSCRITOS ROJOS COMPONIENDO UN PAREADO A JUEGO. ABAJO: RAMA FLORECIENTE FLANQUEADA POR UN PAREADO A JUEGO. SOBRE ELLA HAY UNA PLACA DE MADERA CON UNA CALIGRAFÍA DE LA TORRE DE LA LARGA TARDE DE ZHENG.

Todas las fotografías de esta página son cortesía de R. Mason.
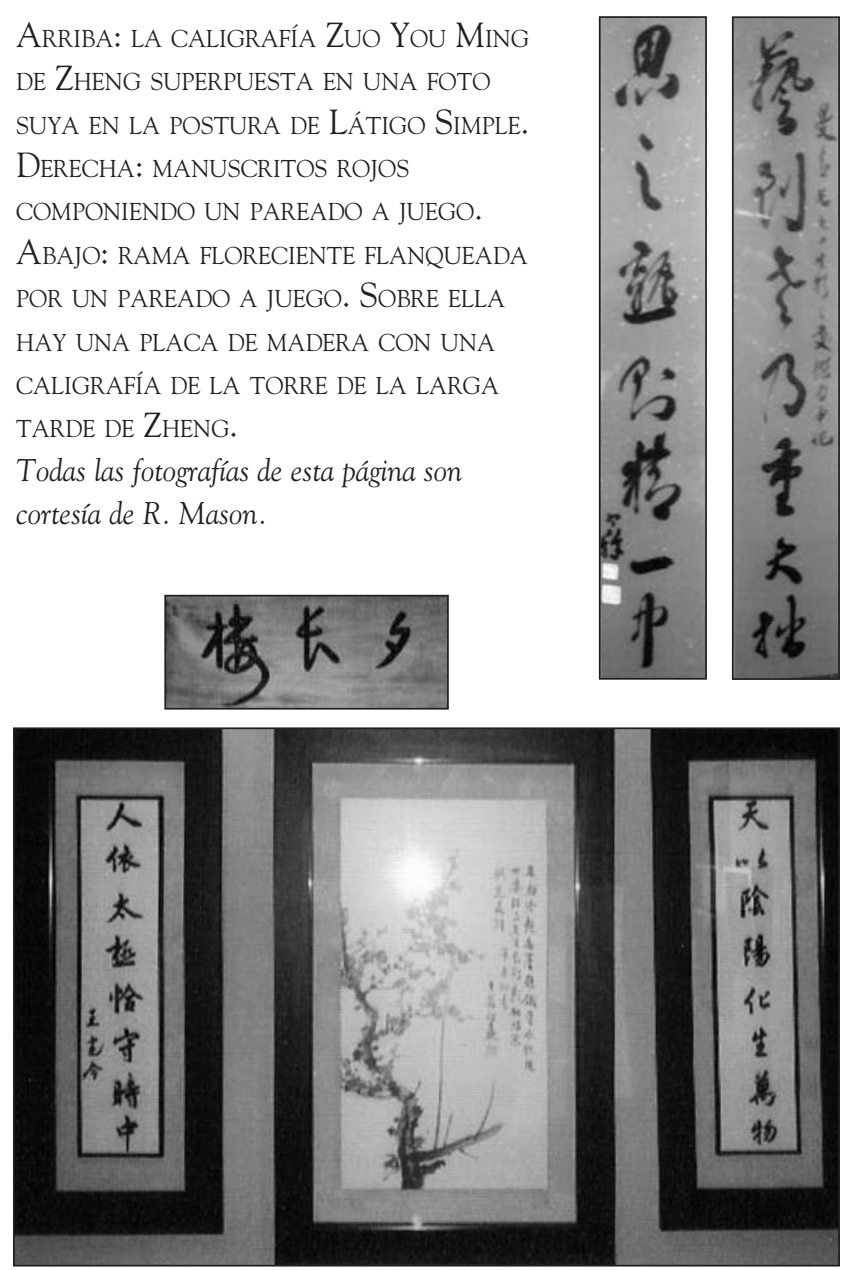

\section{El Zuo You Ming de Zheng Manqing}

Cuando uno entra por la puerta principal del Salón Conmemorativo, justo delante y hacia la pared izquierda, hay un gran panel mostrando una fotografía de 1949 de Zheng en la postura de Látigo Simple. Superpuesta en la foto esta la caligrafía zuo you ming. Los términos zuo you se traducen literalmente como "mano derecha asentada" y ming puede ser interpretado como "inscripción", "lápida", o "lema". Por tanto, la frase "zuo you ming" podría referirse a algo que es grabado y mantenido en la mano cerrada para ser una referencia constante, como en "palabras para vivir". Escrito en un estilo escueto, clásico, los versos del poema de Zheng resuenan con insinuación literaria y podrían ser interpretados de varias formas. Lo esencial del lema dice algo así como:

Deja que las manos vayan ligeras y humildes;

Deja que los pies sean pesados, avanzando con dignidad. ¡Mantente erguido, mantente erguido! Estate sereno y recto. Habla con moderación, con franqueza, y con gran atención; Se compasivo y generoso: lejos de un corazón centrado, regresa.

Se fiel, tolerante, y paciente en tus relaciones;

Deja que tus actitudes hacia otros sean moderadas.

Arrepiéntete, y corrige tus errores con una meditación silenciosa.

\section{Manuscritos rojos con pareado a juego}

Arriba, en ambos lados de la entrada, entre la sala de conferencias y la oficina del Profesor Zheng, cuelgan verticales dos manuscritos rojos de poesía. La pareja constituye un poema de una estructura especial llamada duilian. En tales manuscritos emparejados, la redacción y la gramática china tienen que ajustarse perfectamente; por lo tanto, la creación de este tipo de verso es muy exigente. Las dos secciones del pareado mostrado aquí rezan algo así como:

\section{Cuando reflexionas profundamente, alcanzarás una alta competencia en el área de tu destreza especial.}

\section{Cuando tu habilidad artística ha alcanzado la madurez, sondearás las profundidades de la gran simplicidad.}

\section{Pareado a juego con rama floreciente}

Detrás del escritorio del Profesor Zheng cuelga una pintura de una rama floreciente. Sobre la pintura hay una placa de madera con la inscripción: "Torre de la Larga Tarde" ( $X_{i}$ Chang Lou), el apodo de Zheng para su estudio. En ambos lados de la pintura hay otro pareado. El pareado dice:

El cielo creó todas las cosas vivientes del yin y el yang.

Toda la humanidad depende del Taiji para proteger el Shi Zhong. 


\section{El Pasillo de la Felicidad}

En esta obra de caligrafía ancha y horizontal de caligrafía, la preciosa pincelada del Profesor Zheng muestra su poema: "El Pasillo de la Felicidad" (Tang Le Kuai). El texto del poema aparece debajo tal y como lo traduce Tam Gibbs:

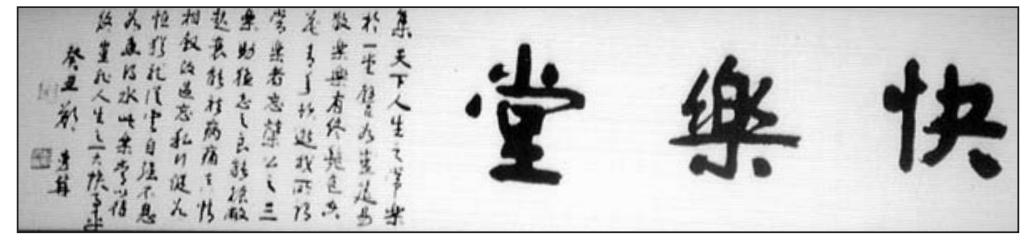

Caligrafía del Pasillo de la Felicidad (Tang Le Kuai).
Podría la alegría que es eterna juntarse en este pasillo. No la alegría de una fiesta suntuosa, que se escurre incluso cuando dejamos la mesa; ni lo que la música trae -es sólo de una duración limitada. La belleza y una cara bonita son como las flores: florecen durante un momento, luego mueren. Incluso nuestra juventud se escabulle rápidamente y se va. No; la felicidad duradera no está en eso, ni en las tres alegrías de Tung Kung. También podemos olvidarlas, la alegría que quiero expresar esta a mundos de distancia. Es la alegría del crecimiento continuo, de la ayuda a desarrollar en nosotros mismos y en otros los talentos y aptitudes con las que nacemos -los regalos del cielo a los hombres mortales. Es restablecer lo extenuado y rejuvenecer aquello que está en declive de modo que somos capaces de disipar la enfermedad y el sufrimiento. Deja al verdadero cariño y a la felicidad confluir habitando en este pasillo. Déjanos aquí corregir nuestros errores pasados y perder la preocupación en nosotros mismos. Con la constancia de los planetas en sus órbitas o del dragón en su camino envuelto de nubes, déjanos entrar en la tierra de la salud y caminar siempre dentro de sus límites. Deja que nos fortalezcamos contra la debilidad y aprender a ser independientes, sin un momento de lapso. Entonces nuestra determinación se convertirá en el preciado aire que respiramos, el mundo en que vivimos; luego seremos tan felices como un pez en aguas cristalinas. Esta es la alegría que perdura, la que podemos llevar con nosotros mismos hasta el final de nuestros días. Y dime, si puedes: ¿Qué felicidad más grande puede ofrecer la vida?

\section{La caligrafía estilizada de Ying Erpo de Zheng Kung}

Colgando en la sala más grande de la planta baja, a la izquierda de la vitrina, hay una obra de arte especial creada por Ying Erpo. Consiste en un carácter estilizado para el nombre familiar "Zheng" en la forma de una pintura del Profesor Zheng preparándose para ejecutar la postura Cuerpo Girado y Golpe de Loto con la Pierna. La figura es como una adivinanza, con varias partes del cuerpo compuestas de pinceladas con temperamento. La inscripción a la derecha de la imagen dice:

\section{El fundador del Taijiquan Zhang Shanfeng. El carácter del nombre del verdadero sucesor, el Maestro Zheng Manqing, está aquí estilizado en esta figura en memoria de la inauguración del Salón Conmemorativo Zheng Manqing en este 25 de junio (cumpleaños de Zheng) del año 2006.}

La inscripción de abajo a la izquierda dice:

Dibujado respetuosamente por Ying Erpo.

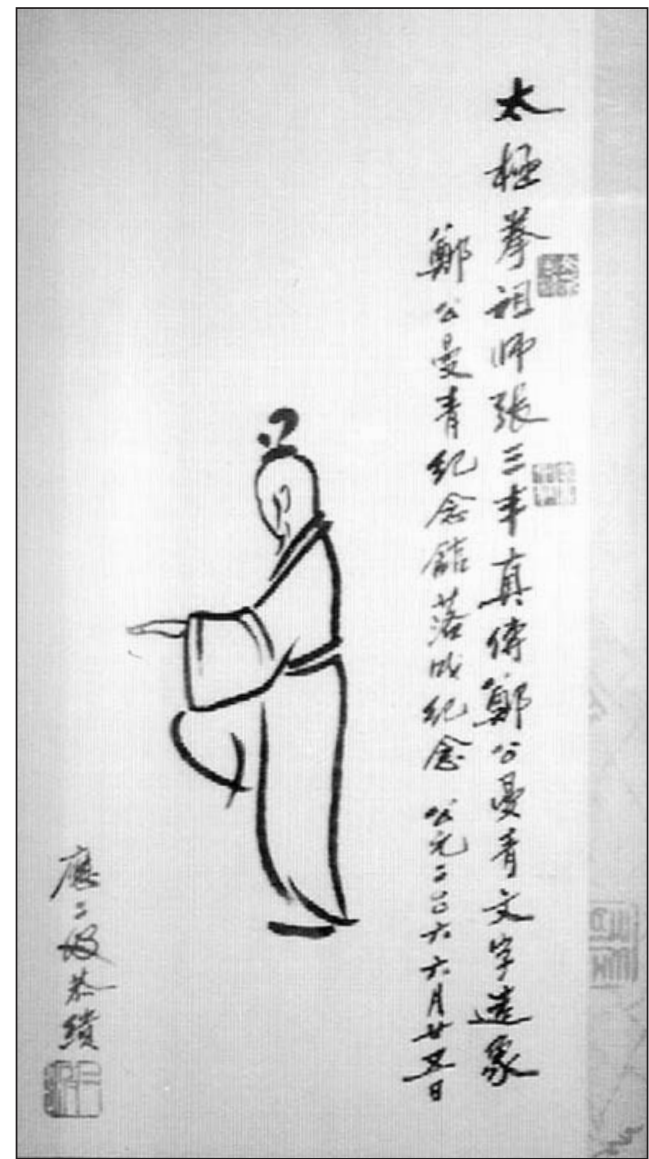

Fotografía de R. Mason.

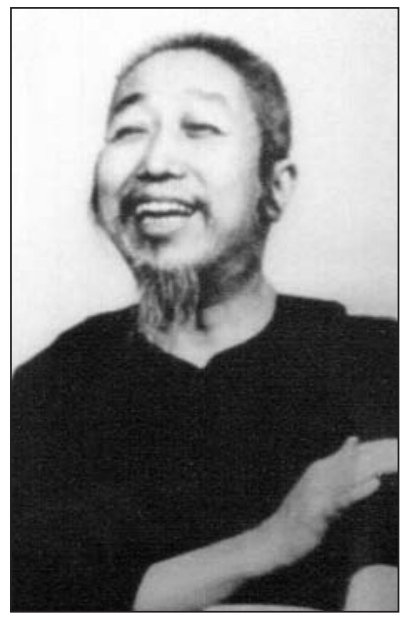

El PROFESOR ZHENG

EXPLICA CON UNA SONRISA
El DibUjO ESTILIZADO DE YING ERPo DEL CARÁCTER "ZHENG".

Fotografía de R. Mason. 


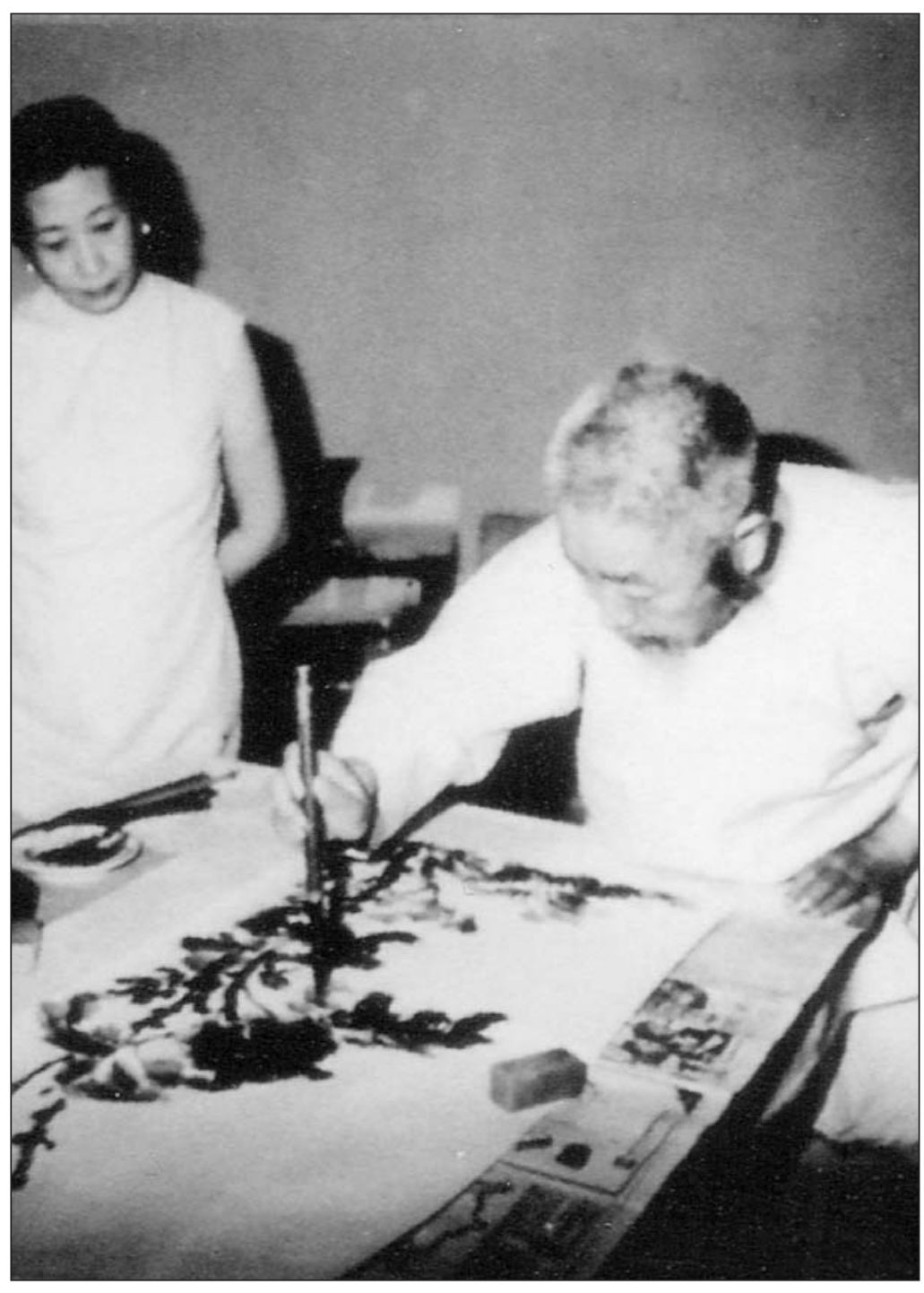

ARRIBA: EL PROFESOR

ZHENG DA PINCELADAS

MIENTRAS MADAME ZHENG

OBSERVA. Cortesía del Salón

Conmemorativo Zheng Manqing.

DERECHA: UN SENCILLO LOTO PINTADO POR EL PROFESOR ZHENG.

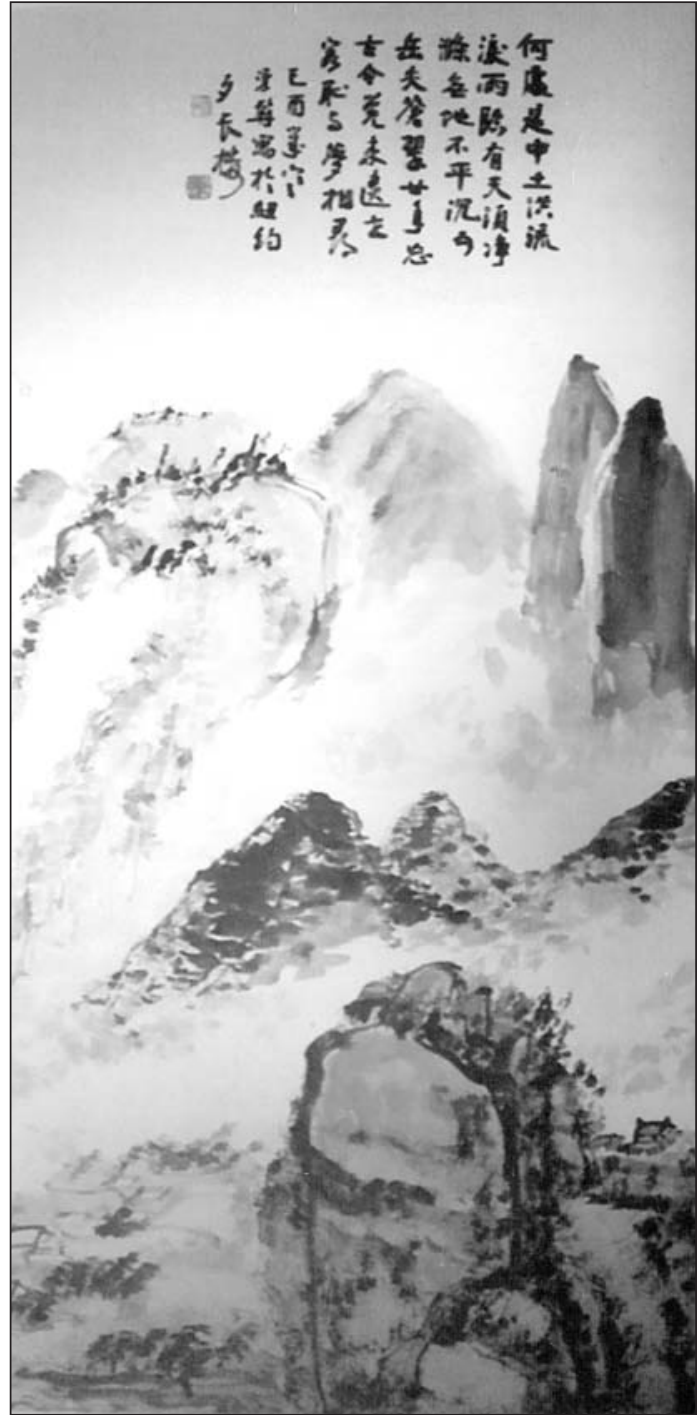

ARRIBA: UN EJEMPLO DE PAISAJE DE MONTAÑA DEL PROFESOR ZHENG.

Debajo: Zheng DESCANSANDO.

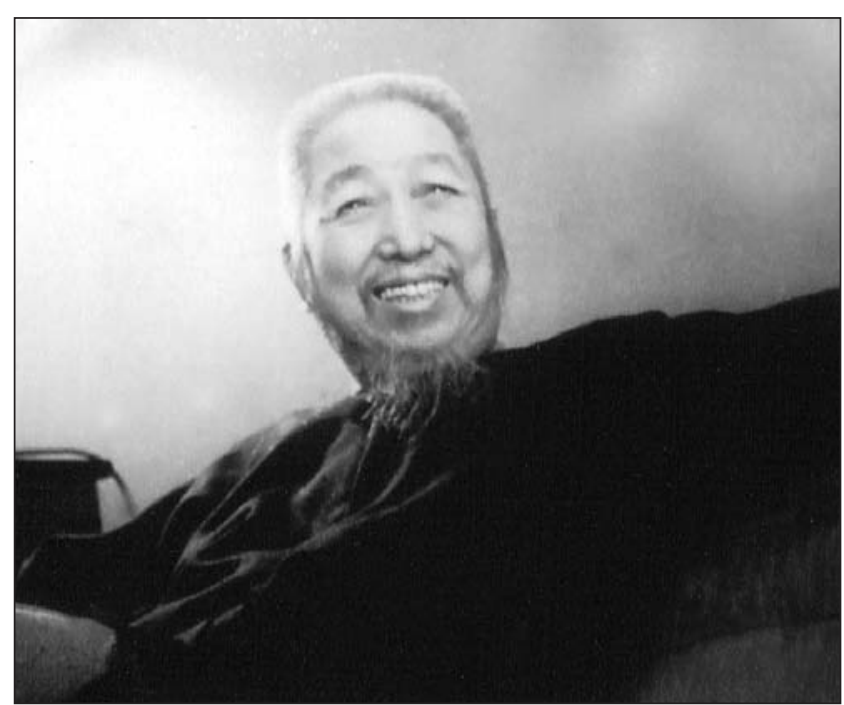



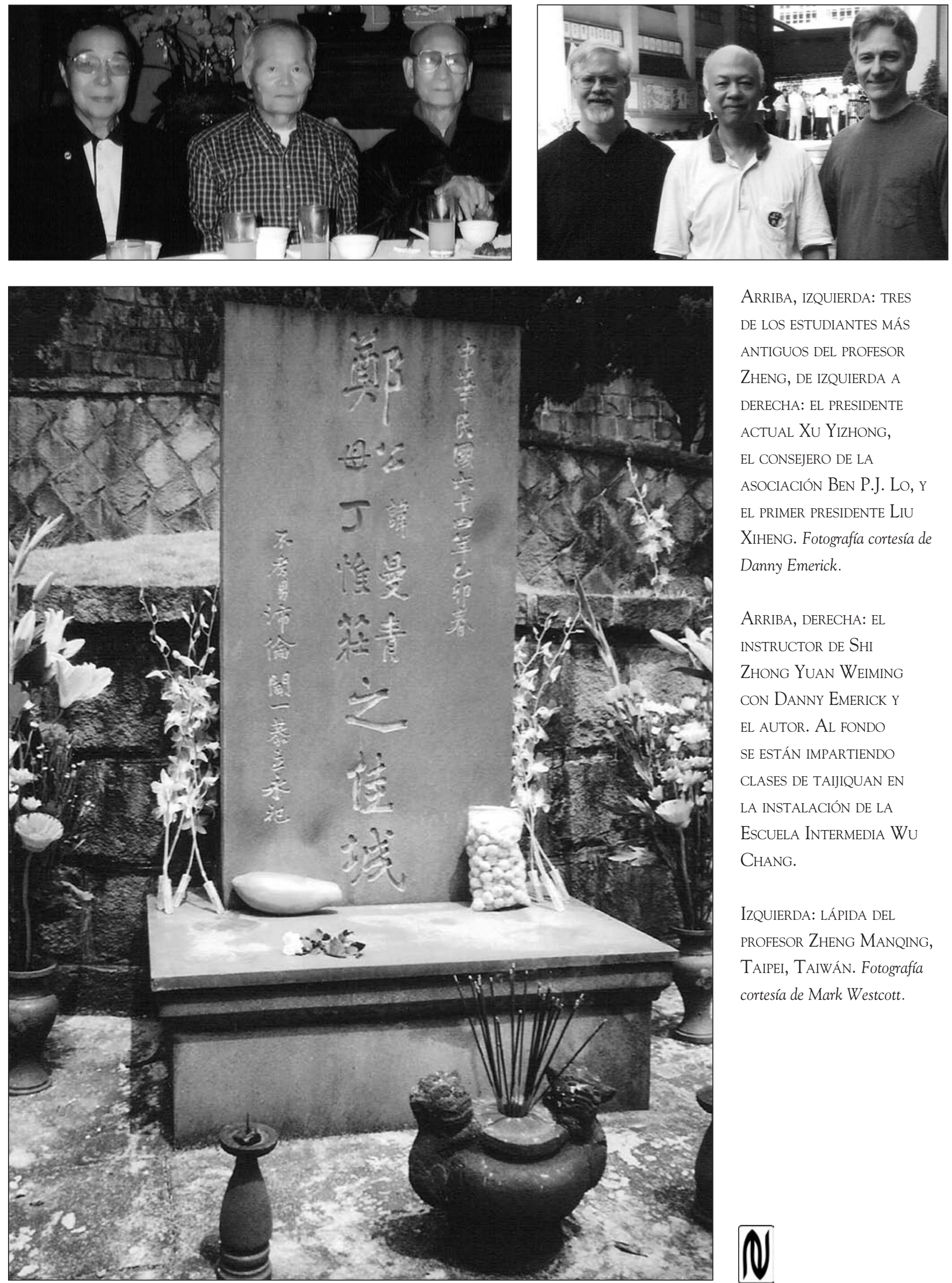

ARribA, IZQUIERDA: TRES DE LOS ESTUDIANTES MÁS ANTIGUOS DEL PROFESOR ZHENG, DE IZQUIERDA A DERECHA: EL PRESIDENTE actual Xu Yizhong, EL CONSEJERO DE LA ASOCiación Ben P.J. Lo, y EL PRIMER PRESIDENTE LIU XiHeng. Fotografía cortesía de Danny Emerick.

ARribA, DERECHA: EL INSTRUCTOR DE SHI ZhONG YUAN WeIMING CON DANNY EMERICK Y EL AUTOR. Al FONDO SE ESTÁN IMPARTIENDO CLASES DE TAIJIQUAN EN LA INSTALACIÓN DE LA ESCUEla INTERMEDIA Wu CHANG.

IZQUIERDA: LÁPIDA DEL PROFESOR ZHENG MANQING, TAIPEI, TAIWÁN. Fotografía cortesía de Mark Westcott.

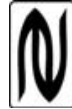


GLOSARIO

\begin{tabular}{|c|c|c|c|c|c|}
\hline Zheng & & Chen Zhicheng & 陳至誠 & Shi Zhong Xueshe & 時中學社 \\
\hline Manqing & & Chen Panling & 陳泮嶺 & Tang Le Kuai & 堂樂快 \\
\hline Jinian & & Chen Weiming & 陳微明 & Tao Bingxiang & 陶炳祥 \\
\hline Guan & & duilian & 㭙聯 & tuishou & 推手 \\
\hline & & Fu Zhongwen & 傅鍾文 & Xiong Yanghe & 熊養和 \\
\hline & & guanxi & 關係 & Xu Yizhung & 徐憶中 \\
\hline & & Li Yaxuan & 李雅軒 & Yang Chengfu & 楊澄甫 \\
\hline & & Liang Dongcai & 梁棟材 & Yang Jianho & 楊健候 \\
\hline & & Lin Sen & 林森 & Yang Shaohou & 楊少侯 \\
\hline & & Liu Xiheng & 劉錫亨 & Yonghe City & 永和市 \\
\hline & & Lo Ben & 羅邦楨 & Zheng Manqing & 鄭曼青 \\
\hline & & neigong & 內功 & Zheng Yue & 鄭岳 \\
\hline
\end{tabular}

\section{NOTAS}

1 Aunque Yue fue el nombre de pila, más tarde Zheng adoptaría el apodo Manqing. Otros apodos y títulos le fueron concedidos por otros, y adoptó diversos seudónimos. Véase los "recuerdos de Zheng" de Yan Changkong (Wile, 2007: 32), los comentarios de Min Xiaoji en su breve biografía del "Man-Jan" (Cheng, 1985: 13-15), y Barbara Davis (1996: 39) para más detalles biográficos.

2 El profesor asistente y traductor americano del Profesor Zheng, Tam Gibbs, proporciona detalles en la breve biografía de Zheng añadida a su traducción del comentario de Zheng sobre Laozi (Cheng, 1971: 237). Barbara Davis cita el Gran Diccionario de Nombres y Alias de la China Contemporánea y Moderna como fuente de detalles sobre Wang Xiangchan, cuyo nombre de pila era Ruyuan y quien era conocido por sus pinturas de flores y plantas, la especialización que sería el punto fuerte de Zheng (Davis, 1996: 39, 53).

3 Esta historia le fue contada al autor por Benjamín P.J. Lo en una conversación el 29 de abril de 2007. También apareció una versión de la historia en un artículo de Xu Yizhong, traducido al inglés por Douglas Wile (2007: 29).

4 Véanse los comentarios de Liang Dongcai registrados por Robert W. Smith (1974: 29) y en las propias escrituras del Profesor Zheng sobre su recuperación de una enfermedad de pulmón (Cheng, 1985: 64).

5 Véanse los comentarios de Yan Changkong (Wile, 2007: 32).

6 Zheng analiza su temprana y esporádica práctica así como su perseverancia y máxima entrega en su libro (Cheng, 1962: 25), e igualmente en su prefacio a la Esencia y Aplicaciones del Taijiquan de Yang Chengfu (Yang, 1934/2005: 1-2).

7 El hecho de que Zheng trabajase con Yang Chengfu, transcribiendo las palabras del maestro para su opus literario final, ha sido agradecido por los hijos de Yang, así como por otras fuentes independientes. Gu Liuxin, el presidente de la Asociación de Artes Marciales de Shanghai, cita el hecho de la contribución de Zheng en su introducción al libro de Yang Zhendou sobre el Estilo Yang de taijiquan (Yang, 1988: 8). Los prólogos escritos por Yang Shouzhong y Zheng Manqing, así como una discusión de las contribuciones de Zheng a la redacción y presentación de las enseñanzas de Yang Chengfu, pueden leerse en la traducción inglesa de Louis Swaim de 2005 de la obra maestra de Yang (Yang, 1934/2005). Véase también Mason (2006) para una revisión 
de la traducción de Swaim. La edición taiwanesa del libro de Yang Zhengguo, Tai Chi Estilo Yang Explicado con Claridad, contiene un prefacio del estudiante de Zheng, Xu Yizhong.

8 Véase el trabajo de Chen de 1929, traducido al inglés en 1985 por Benjamín P.J. Lo y Robert W. Smith, en el que Chen presenta las enseñanzas de su maestro, Yang Chengfu (Chen, 1929/1985: 11-12).

9 Como Chen Weiming, Zheng insistió que en el taijiquan real uno no tiene que utilizar ni siquiera la más ligera fuerza, uno tiene que tener una fe total en el principio de relajar el cuerpo y la mente totalmente (Cheng y Smith, 1967: 101). Siguiendo a Yang Chengfu, Chen Weiming también escribió que aquellos que insisten en utilizar la fuerza nunca obtienen la esencia del taijiquan: "No pueden creer que en el límite de la suavidad se encuentra una calidad diferente de fuerza" (Chen, 1929/1985: 18). Yang Chengfu decía continuamente a Zheng que tenía que relajarse completamente, sin utilizar la más ligera fuerza para defenderse. Sólo "invirtiendo en la pérdida" de esta manera, uno puede manifestar la máxima de Laozi de que: "Lo suave y flexible vencerá a lo duro y fuerte" (Cheng, 1985: 22, 87-88). El énfasis de Yang Chengfu en la suavidad reitera las enseñanzas de su padre, Yang Jianhou: “... el primer paso para aprender las aplicaciones marciales del taijiquan es aprender cómo ser ligero y ágil. Eso significa sin fuerza muscular" (Yang, 2001: 3), lo que sucesivamente repite las palabras de los Clásicos del Taiji: "Lo más suave se convertirá en lo más fuerte" (Lo/Inn/Amacker/Foe, 1979: 19, 32, 37, 46, etc.).

${ }^{10}$ En una entrevista (Mason, 2001), Robert W. Smith apuntó que, en su experiencia personal con los exponentes del taijiquan, no había nadie igual a Zheng. Smith se apresuró a añadir, sin embargo, que diciendo esto no estaba manteniendo que Zheng fuera el mejor en China. Como el mismo Zheng reconoció, otros incluso dentro del círculo de Yang Chengfu tales como Li Yaxuan y Chang Qinlin eran superiores a él en el tuishou (Mason, 2001, Vol. 10, no 1: 42). Para informes de los retos de Zheng, véanse las escrituras de Zheng, así como artículos y libros de otros, particularmente de Liang Dongcai, Robert W. Smith, y Douglas Wile. Liang Dongcai reivindica haber estudiado con quince profesores diferentes de taijiquan, incluyendo a estudiantes directos de Yang Chienhou, Yang Banhou, y Yang Shaohou, aunque, como Smith, Liang mantuvo que Zheng era el maestro más grande de taijiquan con el que se había encontrado personalmente. Otras evidencias apoyan la legitimidad de las habilidades de Zheng (véanse Hayward, 1993: 94; Wile, 2007: 24-25; Smith, 1974/1990: 37-42; Smith, 1999: 196-197, 304; Smith, 1995, Vol. 4, no 3, etc.).

${ }^{11}$ Zheng comenta sus razones para abreviar la forma de la familia Yang en su trabajo "Taijiquan y Educación Física", en la sección: "En las artes marciales se busca la calidad y no la cantidad" (Wile, 2007: 103-104, véase también la p. 80) y también en su trabajo de 1965 (Cheng, 1965/1999: 9). La utilización de Zheng del número 37 es notable y no sin precedentes. Chen Weiming, en su texto de 1929, seleccionó fotografías de varias posturas importantes para la práctica, citando como precedente que: "Hace mucho tiempo [Xu Xianbing] enseñó un ejercicio de 37 posturas del Taijiquan estilo Yang en su canción: "Nueve secretos clave del taijiquan" (Yang, 2001: 7). [Xu Longhou] ofrece una teoría sobre el origen del taiji en el que había un ermitaño llamado [Xu Xuanbing] que vivió en la provincia de Anhuei durante la Dinastía Tang y creó un estilo de taijiquan llamado "Treinta y siete", después de los movimientos de su forma (véase Smith, 1975/1990: 115; y Jou, 1981/1991: 8-9). Aunque Zheng no explica el significado del número, uno podría asumir que es digno de atención ya que aparecen varias listas de posturas en los libros escritos por Zheng, aunque el número total siempre asciende a 37 (véase la introducción del traductor, Cheng, 1965/1999: x).

12 Véase la entrevista informativa de Davis y Mann (1996) con Benjamín P.J. Lo, el primer estudiante del Profesor Zheng en Taiwán, y abanderado en los Estados Unidos. 
${ }^{13}$ El nombre Shi Zhong (Shih Chung, como fue romanizado por primera vez en Taipei, o Shih Jung como fue transliterado más tarde por la rama americana en la ciudad de Nueva York) se refiere a las ideas de ritmo y equilibrio correcto y resuena con las alusiones literarias de la Doctrina del Medio y otros clásicos de la literatura y filosofía china tradicional, en las que Zheng era experto.

${ }^{14}$ Véase el texto clásico de Robert W. Smith (1974/1990), y la siguiente autobiografía (1999), para detalles de las aventuras del pionero historiador de las artes marciales en Taiwán. Véase también Mason (2001) para una extensa entrevista con Smith.

${ }^{15}$ Para más detalles véase la "Breve biografía del profesor M.C. Cheng" del traductor Tam Gibbs en el comentario del Profesor sobre Laozi (Cheng, 1971/1981: 239).

${ }^{16}$ De acuerdo con una declaración que circula por Internet de Bill Law de la Asociación para el Estudio del Cheng Zi Taichi Chuan de Melbourne (2/15/02), Ju Hongbin, quien da clases en gran parte del sur de Taiwán así como en el Shi Zhong de Taipei, dedicó un Salón Conmemorativo separado a la memoria del Profesor Zheng en Gaoxiong el 23 de septiembre de 2001. El piso esta situado en la Calle Ming Hua 255, 2/F en el distrito Gu Shan de Gaoxiong.

${ }^{17}$ En 1982 el Museo del Palacio Nacional, en cooperación con Madame Zheng, presentó una exhibición retrospectiva especial de más de dos docenas de pinturas y trabajos caligráficos del Profesor Zheng. Esto fue un honor extraordinario, ya que rara vez el museo ofrece trabajos de artistas modernos. Se publicó un catálogo de grabados junto con un prefacio escrito previamente por Madame Chiang Kai-shek, quien había sido una estudiante de pintura del Profesor Zheng (Davis, 1996: 48).

${ }^{18}$ Para más información véase la "Entrevista con el gran maestro Hsu Yee Chung" editada por Maximiliano Biondi y publicada en la página web de la Sociedad de Estudio Shi Zhong en http://www.37taichi.org.tw/

${ }^{19}$ Pueden encontrarse más detalles de la ceremonia de apertura en el librillo Registro de Donantes del Salón Conmemorativo Zheng Manqing, publicado por la Asociación en 2007.

\section{AGRADECIMIENTOS}

Los agradecimientos son para todos aquellos que contribuyeron en este trabajo en pequeña o gran medida. Xu Yizhong ofreció su apoyo entusiasta y su permiso cortés para utilizar las fotografías y materiales. Benjamín P. J. Lo, Robert W. Smith, Liu Xiheng, Yuan Weiming, y Danny Emerick proporcionaron fotografías y otra ayuda, como hicieron Monica Chen, Julia Fairchild, Ken Van Sickle, Mark Westcott y otros. Se tiene una deuda especial de gratitud con Nick Tan y Jeff Herrad (así como a otros investigadores referenciados en la bibliografía) por su ayuda con la traducción de las fuentes. Se agradecen las correcciones de los lectores. Al autor le gustaría agradecer al difunto Tam Gibbs y a Ed Young y Maggie Newman su ayuda y ánimos. Para más información sobre la Asociación para el Estudio del Taijiquan del Maestro Zheng, por favor póngase en contacto a través de su página web www.37taichi@yahoo.com.tw.

\section{BIBLIOGRAFÍA}

BARNSTONE, T. y CHOU, P. (1996). The art of writing: Teachings of the Chinese masters. Boston: Shambala.

BIONDI, M. (2006). Interview with grand master Hsu Yee Chung. Publicada en la página web de la Sociedad de Estudio Shi Zhong en: http://www.37taichi.org.tw/

CHEN, W. (1929/1985). T'ai chi ch'uan ta wen: Questions and answers on t'ai chi ch'uan (traductores B. Lo y R. Smith). Berkeley, CA: North Atlantic Books. 
CHENG, M. (1962). T'ai chi ch'uan: A simplified method of calisthenics for health and self defense. Taipei: Shi Zhong Taijiquan Center.

CHENG, M. (1965/1999). Master Cheng's new method of taichi ch'uan self-cultivation (traductor M. Hennessy). Berkeley, CA: Frog, Ltd.

CHENG, M. y SMITH, R. (1967/2004). T'ai chi. Rutland, VT: Charles E. Tuttle.

CHENG, M. (1971/1981). Lao-tzu: My words are very easy to understand (traductor T. Gibbs). Berkeley, CA: North Atlantic Books.

CHENG, M. (1985). Cheng tzu's thirteen treatises on t'ai chi ch'uan (traductores B. Lo, y M. Inn). Richmond, CA: North Atlantic Books.

CHENG, M. (1996). T'ai chi ch'uan: A simplified method of calisthenics for health and self defense. [Vídeo]. Ashville, NC: Cho San.

CHENGTZU TAI-CHI CHUAN RESEARCH ASSOCIATION, (2007). Cheng Manch'ing Ji Nien Guan Doners' Record. Taipei, Taiwan.

DAVIS, B. (1996). In search of a unified dao: Zheng Manqing's life and contributions to taijiquan. Journal of Asian Martial Arts, 5(2), 36-59.

DAVIS, D. y MANN, L. (1996). Conservator of the taiji classics: An interview with Benjamin Pang Jeng Lo. Journal of Asian Martial Arts, 5(4), 46-67.

FAIRCHILD, J. y LIN, G. (2007). The beginnings, growth, and development of taijiquan in Taiwan: An interview with chairman Wang Yennien. Tabei, Taiwan: Yen-nien Shanghao, publicada en el sitio web Yen-nien Daoguan.

HAYWARD, R. (1993). T'ai-chi ch'uan: Lessons with master T. T. Liang. St. Paul, MN: Shukuang Press.

JOU, T. (1981/1991). The tao of tai-chi chuan: Way to rejuvenation. Warwick, NY: Tai Chi Foundation.

LO/INN/AMACKER/FOE (1979). The essence of t'ai chi ch'uan: The literary tradition. Richmond, CA: North Atlantic Books.

MASON, R. (2001). Fifty years in the fighting arts: An interview with Robert W. Smith. Journal of Asian Martial Arts, 10(1), 36-73.

MASON, R. (2006). Revisión del libro Yang Chengfu: The essence and applications of Taijiquan. Journal of Asian Martial Arts, 15(3), 92-93.

SMITH, R. (1974/1990). Chinese boxing: Masters and methods. Berkeley, CA: North Atlantic Books.

SMITH, R. (1975). A master passes: A tribute to Cheng Man-ch'ing. Shr Jung Newsletter, $1(1), 2-7$.

SMITH, R. (1995). Remembering Zheng Manqing: Some sketches from his life. Journal of Asian Martial Arts, 4(3), 46-59.

SMITH, R. (1999). Martial musings: A portrayal of martial arts in the 20th century. Erie, PA: Via Media.

WILE, D. (1985). Cheng Man-Ch'ing's advanced t'ai-chi form instructions. Brooklyn, NY: Sweet Ch'i Press.

WILE, D. (2007). Zheng Manqing's uncollected writings on taijiquan, qigong, and health, with new biographical notes. Milwaukee, WI: Sweet Ch'i Press.

YANG, C. (1934/2005). Yang Chengfu: The essence and applications of taijiquan (traductor L. Swaim). Berkeley, CA: North Atlantic Books.

YANG, J. (2001). Tai chi secrets of the Yang style. Boston, MA: YMAA Publication Center.

YANG, Z.D. (1988). Yang Style Taijiquan. Hong Kong: Hai Feng Publishing Co. and Beijing, China: Morning Glory Press.

YANG, Z. (1993). Yang Cheng Fu shi tai ji quan. Guangxi Province, China: Guangxi Minzu.

YU, W. y SHARP, G. (1993). Fu Zhongwen: A Yang family legend. Inside Kung-fu, Abril 1993, 44-46. 